\title{
Workshop on Stellar Tidal Disruption
}

\author{
Organized by Glennys R. Farrar \\ Center for Cosmology and Particle Physics, New York University, USA \\ email: gf25@nyu.edu
}

\begin{abstract}
The past year has seen major advances in the observational status of Stellar Tidal Disruption, with the discovery of two strong optical candidates in archived SDSS data and the real-time X-ray detection of Swift J1644+57, plus rapid radio and optical follow-up establishing it as a probable Tidal Disruption Flare (TDF) in "blazar mode". These observations motivated a workshop devoted to discussion of such events and of the theory of their emission and flare rate. Observational contributions included a presentation of Swift J2058+05 (a possible second example of a TDF in blazar mode), reports on the late-time evolution and X-ray variability of the two SwIFT events, and a proposal that additional candidates may be evidenced by spectral signatures in SDSS. Theory presentations included models of radio emission, theory of light curves and the proposal that GRB101225A may be the Galactic tidal disruption of a neutron star, an interpretation of Swift J1644+57 as due to the disruption of a white dwarf instead of main-sequence star, calculation of the dependence of the TDF rate on the spin of the black hole, and analysis of the SDSS events, fitting their SEDs to profiles of thoretical emission from accretion disks and showing that their luminosity and rate are consistent with the proposal that TDEs can be responsible for UHECR acceleration.
\end{abstract}

Keywords. Tidal Disruption, Black Hole, Flare, AGN, SNe

\section{Overview}

This workshop, organized by G.R. Farrar and attended by about 30 people, occupied two afternoon sessions. The first focussed on observations, with contributions by S. van Velzen, A. Levan, A. Zauderer, B. Cenko, and S. Komossa, plus predictions for radio emission by B. Metzger and S. van Velzen. The second session was more theoretical, beginning with theories of emission (G. Lodato, E. Rossi and T. Piran) and continuing with rate and modelling (M. Kesden and G. Farrar). There was very lively discussion throughout. This summary emphasizes material that was not presented elsewhere in the Symposium.

\section{Observations of Probable Tidal Disruption Flares}

\subsection{Tidal Disruption Events in SDSS Stripe 82}

Van Velzen described the results of van Velzen et al. (2011), in which two probable tidal disruption events (TDE) were identified in archived SDSS "Stripe 82" data. Stripe 82 is a $300-\mathrm{deg}^{2}$ region in which 2.6 million galaxies were observed on average 70 times each, over eight years. The identification pipeline minimized contamination from variable AGNs by excluding hosts in a QSO colour locus, and from SNe by requiring flares to be nuclear; Fig. 1 (left) shows the fit of the observed separations to nuclear+stellar flares. Requiring 3 detections in $u, g$ and $r$, and also that $d<0^{\prime \prime} .2$, leaves 42 nuclear flares out of 342 in the original sample. All but two of these nuclear flares are removed by eliminating spectroscopic AGN and requiring no variability in the off-seasons. The colours and cooling of these two candidate tidal disruption flares (TDFs) are shown in 

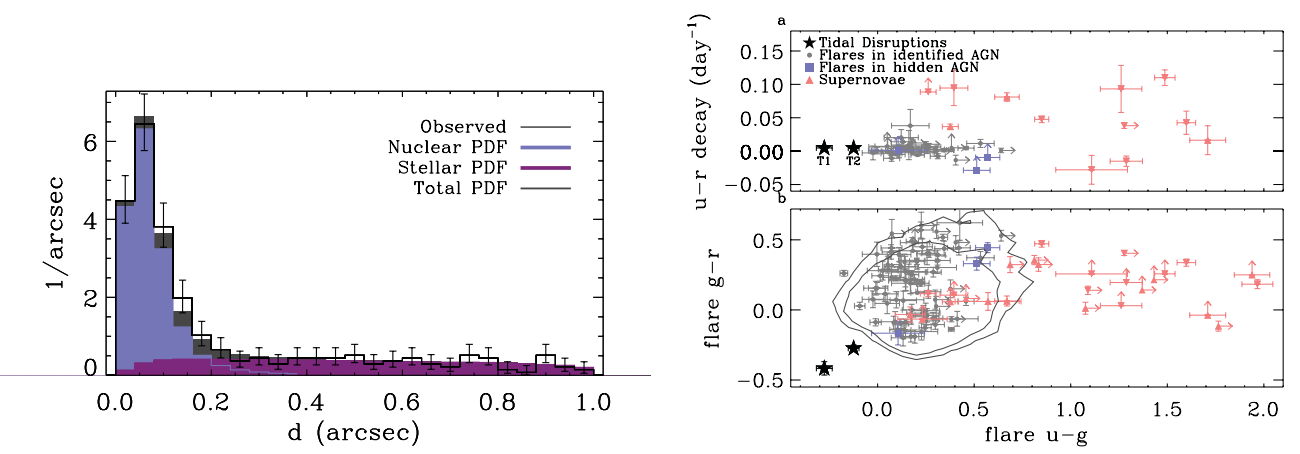

Figure 1. Left: Distribution of flare offsets (van Velzen et al. 2011a). Right: Comparison of candidate TDE flares to other flares in the analysis.

Fig. 1 (right), where it is seen that they are far removed from other AGNs or SNe in Stripe 82. The relative flux increase in the TDF candidates is much larger than has been observed for any other AGNs in the sample, with estimated probabilities of $10^{-7}$ and $10^{-5}$ that they are sampled from the AGN flare distribution. The probability of finding such low off-season variability for AGNs is estimated to be $10^{-6}$ and $10^{-5}$, and the radio emission is $<1 \mathrm{mJy}$ for both. Nor are they explained by being SNe; it is particularly telling that both are detected in the UV more than 2 years after the flare - see Fig. 2.

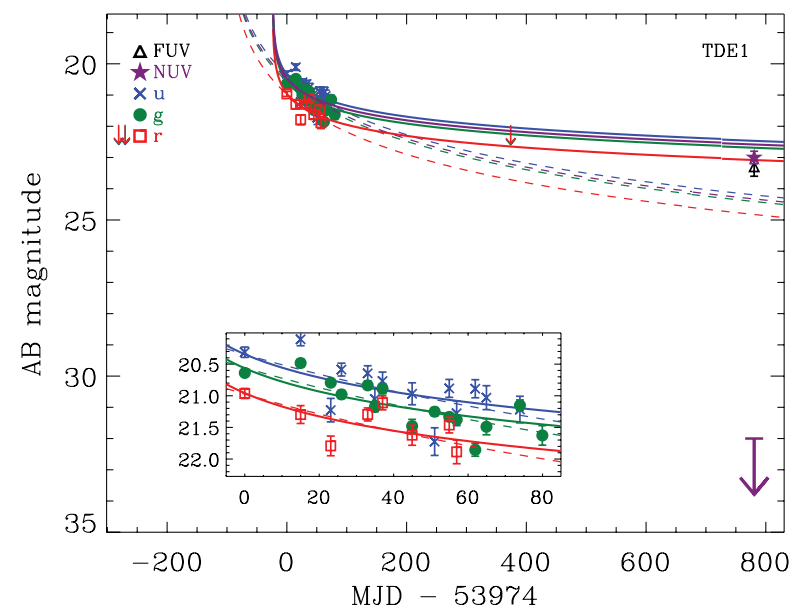

Figure 2. Light curve of TDE1, including the UV detection 800 days after the optical detection. The largest possible UV flux for a SN origin for this flare is shown by the large downward-pointing arrow.

The flaring-state spectrum of TDE2 is unlike any known SN, and the likelihood of it being a Type IIn SN, so close to the nucleus, is $<0.08 \%$. It cannot be ruled out that they are a new kind of "nuclear" core-collapse SN, but that would require a factor 1000 suppression of such events outside the nuclear region. Van Velzen et al. (2011) conclude that they are most likely TDFs, from black holes of mass $\sim 10^{7-8} \mathrm{M}_{\odot}$. Extrapolation from the observed rate and sensitivity suggests that about 10/yr are expected in CRTS, QUEST and Pan-STARRS-1 Medium-Deep Field, and $\sim 4000 / y r$ in LSST. This presentation drew many comments and questions, especially regarding the possibility of a new type of nuclear SN. 


\subsection{Swift J1644 +57 in the Optical and $X$-ray}

Levan reviewed the key optical and X-ray observations of Swift J1644+57; details of those observations can be found at http://adsabs . harvard .edu/abs/2011Sci . . 333 . 199L, -/2011Sci..333..203B and -/arXiv1107.5307, and there was further extensive discussion of that event throughout the Workshop. Levan also reported that, since the publication of that paper, the source had continued a steady overall decline in X-ray emission, but recently more rapid X-ray variability seemed to have become apparent. The IR source continues to fade, while presumably becoming progressively more hostdominated; the IR fading lowers the black-hole (BH) mass estimate (based on the host stellar mass) by a factor of two. There is a low but marginally significant IR linear polarization $(7.4 \pm 3.5 \%)$ about 20 days after the outburst. How can we find more of these events? Where is the best place to search — radio, hard X-ray, soft X-ray? — and can we identify them easily? Are there smoking guns for their origin for which we should be looking? Can the examples that have been found so far still yield new physics from late observations?

\subsection{Swift J1644+57 in the Radio}

Zauderer described the EVLA discovery of radio emission from Swift J1644+57, highlighting that early localization (within a day of the trigger from SWIFT) was consistent with the centre of a normal galaxy at $z=0.354$. Radio observations from $1-345 \mathrm{GHz}$ obtained in the first month after the burst with many facilities (EVLA, CARMA, SMA, AMI Large Array, VLBA, OVRO 40-m) support the conclusion that Swift J1644+57 is a TDE: (a) The positional alignment between the optical image of the galaxy and the VLBA centroid, $0^{\prime \prime} .11 \pm 0^{\prime \prime} .18$, or $0.5 \pm 0.9 \mathrm{kpc}$, is consistent with a nuclear origin. (b) SMA and CARMA verify a rising spectrum: a $\nu^{2}$ behavior all the way to 345 $\mathrm{GHz}$ at early times, not seen in long GRBs. (c) The data can be fitted by synchrotron self-absorption, and the derived parameters show the source expanding relativisticallyconsistent with the Mar 25-28 range for the TDE (Zauderer et al. 2011). Follow-up is continuing (see Berger et al. 2012). She told a fascinating story of the difficulty of having a fast-time response and the serendipity involved in this case, and underlined the importance of knowing clearly, for future events, what signatures to look for at the different wavelengths, since response time is critical.

\subsection{Swift J2058+05}

Cenko discussed a second SwIFT event, J2058+05, which also has characteristics of a TDF (Cenko et al. 2011). It was discovered by the SwIFT BAT as a hard X-ray transient in 4-day all-sky co-added data. Follow-up XRT observations revealed a bright X-ray source, with a faint optical counterpart from GROND and a radio counterpart detected by the EVLA. The host galaxy is at $z=1.19$, giving $L_{X} \sim 2 \times 10^{47} \mathrm{erg} \mathrm{s}^{-1}$ for the flare. There is no sign of AGN activity in the spectrum, and the SED is much bluer than for a blazar. Fig. 3 (upper plot) compares the optical and X-ray luminosities of Swift J2058+05 and Swift J1644+57 to other extreme objects. The table in the lower half of Fig. 3 summarizes the key properties of Swift J2058+05 and Swift J1644+57. HST and Keck observations imply $M_{B H}<10^{7} M_{\odot}$. Cenko pressed for answers to several questions: What are the fundamental characteristics that define this class of sources? With incomplete information, how can we distinguish blazar-mode TDFs from blazars? How can we measure collimation? What fraction exhibits relativistic jets, and why? 


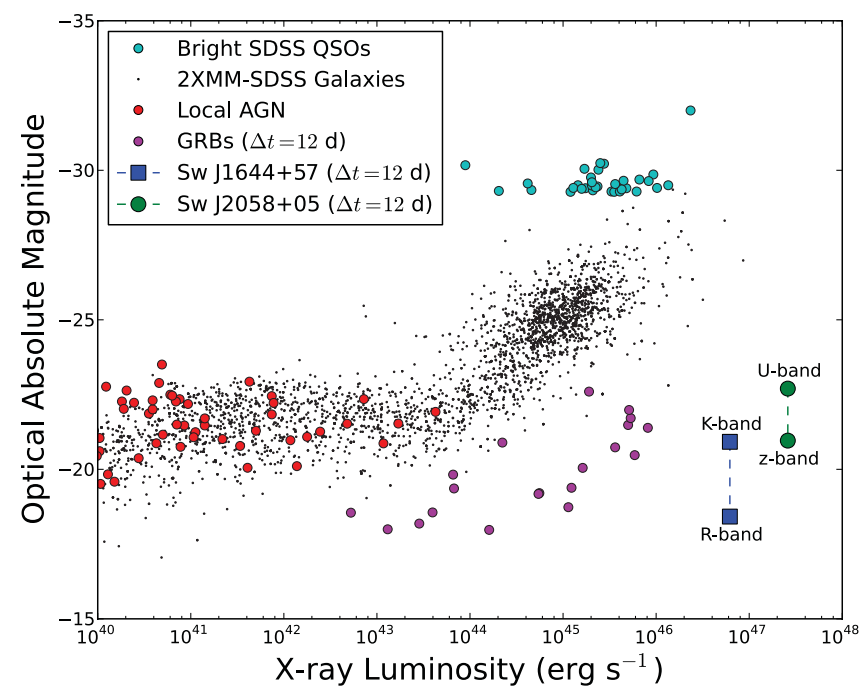

\begin{tabular}{|c|c|c|}
\hline & $\left.S_{W}\right\rfloor 1644+57$ & Sw $\lcm{2058+05}$ \\
\hline Lx peak & $1.00 E+48$ & $3.00 \mathrm{E}+47$ \\
\hline$\alpha_{x}$ & $-1.7(?)$ & -2.2 \\
\hline$\Delta t_{\text {var }}$ & 80 & $<$ IE4 \\
\hline Ex(iso) & $2.00 E+53$ & $3.00 E+53$ \\
\hline$\beta_{\text {ont }}$ & $-1.0(?)$ & 2.0 \\
\hline Mv (transient) & $>-18$ & -21.5 \\
\hline$\beta_{\text {rad }}$ & I.3 & 0 \\
\hline $\mathrm{VL}_{\mathrm{v}}(\mathrm{rad})$ & $1.00 E+4 \mid$ & $1.00 \mathrm{E}+42$ \\
\hline$M_{B H}$ & $<8 \mathrm{e} 6$ & $<$ le 7 \\
\hline$\Gamma$ & 1.3 & $>3$ \\
\hline
\end{tabular}

Figure 3. Upper: Comparison of optical and X-ray luminosities of Swift J2058+05 and Swift J1644+57 to those of other extreme objects. Lower: Properties of Swift J2058+05 and Swift $\mathrm{J} 1644+57$.

\subsection{Tidal Disruption Events from SDSS Spectra}

Komossa reported finding about 5 examples of TDE in SDSS spectra, in which she argues that high-energy ionization lines (of Fe, He) show a light echo from a UV flare. Details will be given in a paper in preparation.

\subsection{Predictions for Radio Emission from TDEs}

Two complementary models of radio emission from tidal disruption events were put forward. Metzger reported a model (Metzger et al. 2011) for the radio afterglows of TDEs, assuming a two-stage jet whose late-time behaviour follows the Blanford-McKee model. The radio emission is expected to peak $\sim 1$ year after a TDE. The radio is beamed, but much less than the X-ray, for Sw1644, according to observations. van Velzen reported a model (van Velzen et al. 2011b) of radio emission based on the jet-disk symbiosis relationship and adopoting an accretion rate from the SDSS TDE flares, but said that the model does not give good representation of the observations of Sw1644. 


\section{Theory and Modelling of Tidal Disruption Flares}

\subsection{Modelling the Light Curves of TDEs}

Lodato reviewed the theory of emission from TDEs. The classic $t^{-5 / 3}$, where $t$ is the time since pericentre passage, describes only the late-time fallback rate; at early times, $t \leqslant 0.2$ years, and the density profile and compressibility of the star produces a range of behaviour. Furthermore, although the bolometric luminosity from the disk (thermal emission) decreases as $t^{-5 / 3}$, a single-wavelength light curve does not exhibit the same scaling. At long wavelengths and early times, the disk thermal emission is in the RayleighJeans tail and $L \sim t^{-5 / 12}$. Attempts have been made (Cannizzo et al. 2011) to model Sw 1644, for which the X-ray emission is dominated by the jet so it is expected to scale with the fallback rate. The early light curve and event rate supports the argument that this was a deeply plunging event with a very short fall-back time of $1-2$ days. However, the later light curve shows a much slower decay, with a fall-back time of $\sim 20$ days that is best fitted with a model for low stellar mass, but it is not clear just how well the model fits. Moreover, GRB101225A has a light curve which can be understood as a Galactic TDE in which a minor object falls onto a neutron star (Campana et al. 2011).

\subsection{Tidal Disruption Model: the Wind}

Rossi discussed super-Eddington mass loss via winds, which obscures the disk emission at early times. The peak is first in the optical region, and then moves to higher frequencies. It is critically important to observe the wind, because super-Eddington accretion is not well understood; observations of the wind, and then of the disk, will therefore give tighter constraints on the physical parameters of the system. Many assumptions are needed for the modelling, so better constraints from observation would be valuable (for instance, to determine that a disk does form in the first place.) The wind should only be important for $M<10^{7} M_{\odot}$, and will be more prominent at optical than UV frequencies. It should show broad absorption lines in the UV (C IV, L $\alpha$, O VI)—-broad and blue-shifted - owing to matter in the wind absorbing photons that are coming from deep in the wind/disk photosphere. The apparent properties of the jet depend strongly on observation angle.

\subsection{Model for J1644+57}

Piran concurred with the general view that J1644+57 is a TDF, but has argued (Krolik \& Piran 2011) that the complex time-structure of its light curve, with multiple time-scales and intensity jumps, constitutes evidence that the disrupted star was a white dwarf rather than a main-sequence star. Specifically, Piran \& Krolik argue for the existence of 3 sub-flares, each lasting about 1000-2000 seconds. Within each there is strong variability on time-scales of 100 seconds: about $3 \times 10^{4}$ seconds between sub-flares, and minima intensities between sub-flares which are suppressed by a factor of 600 compared to the maxima: see Fig. 4 (upper). The interpretation proposed by Krolik \& Piran (2011) is illustrated in Fig. 4 (right): a WD experiences a partial disruption and enters an orbit with period of few $10^{4}$ sec. Each time the WD returns to peri-centre, more material is stripped and a new accretion disk forms. The flare duration of $\sim 1000-2000$ secs is the length of time that the accretion disk takes to drain out. The 100-sec time-scale is interpreted as the characteristic time of the accretion disk. They postulate that the above has a duration of $\sim 2 \times 10^{5}$ sec followed by a gradual decay. The tidal disruption radius for a WD is smaller than for a main-sequence star, owing to its compactness. If the maximum mass of a $\mathrm{BH}$ which can disrupt a main-sequence star without swallowing it whole is $10^{8} M_{\odot}$, the corresponding limit for the disruption of a WD is $\sim 3 \times 10^{5} M_{\odot}$, which seems difficult to accommodate given the observed host galaxy. This proposal 
generated considerable debate, and triggered objections on several grounds, especially the required $\mathrm{BH}$ mass. [Taking into account the results discussed in next contribution from M. Kesden in fact loosens the BH mass constraint.]

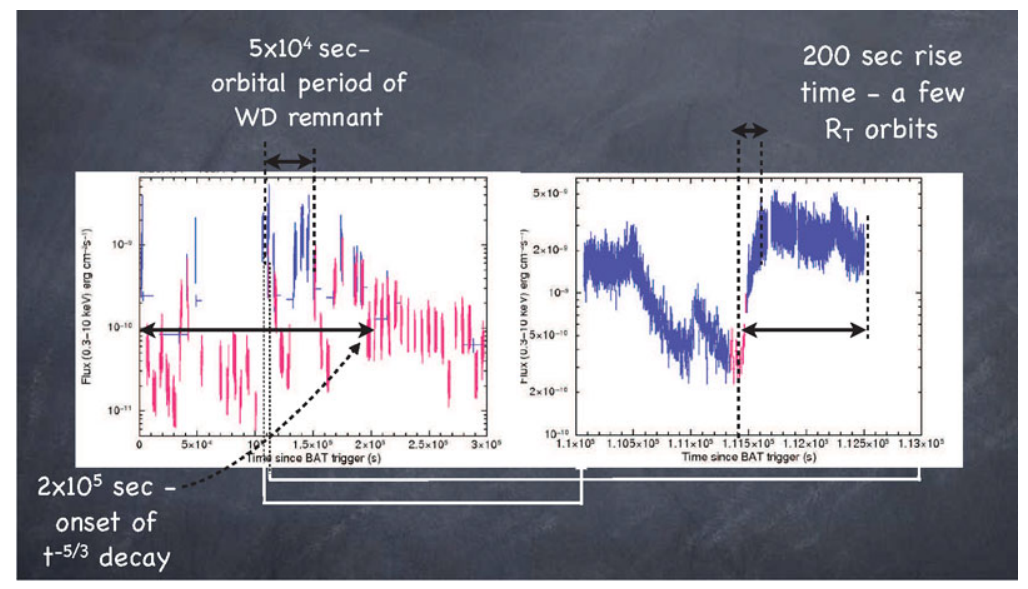

\section{A white Dwarf disrupted by a $510^{5} \mathrm{M}_{\odot}$ black hole - a model for Swift 1644}

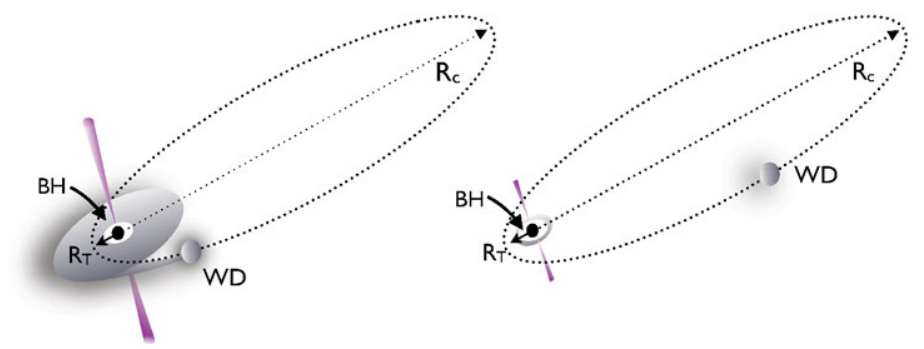

Figure 4. Upper: Sw J1644 light curves. Lower: Piran-Krolik picture of Sw J1644.

\subsection{Tidal Disruption of a Star by a Spinning Black Hole}

Kesden reported recent calculations which show that spinning black holes extend considerably the range of Black Hole masses which produce observable TDFs. Making that change, but otherwise following the rate calculation by Wang \& Merritt (2004), leads to the conclusion that: (a) General Relativity is important for $M_{\mathrm{BH}}>10^{7} M_{\odot}$, (b) the maximum BH mass that can produce an observable TDE increases from $10^{8}$ to $10^{9} M_{\odot}$, and (c) including the reduction in rate due to the existence of direct-capture orbits (applicable even for lower-mass BHs but apparently not previously taken into account) reduces the predicted TDE rate by a factor $2 / 3$ for $10^{7} M_{\odot}$, or $1 / 10$ for $10^{8} M_{\odot}$ (see Fig. 5 ). The upper limit to the $\mathrm{BH}$ mass for the tidal disruption of a white dwarf would increase correspondingly, improving the consistency of the Piran-Krolik model of Sw J1644+57 with the properties of the host galaxy.

\subsection{SDSS TDFs: Rate, Light Curves and UHECR acceleration}

Farrar outlined phenomenological aspects of the SDSS tidal disruption events. The TDE rate inferred from the two observed SDSS TDFs is $10^{-5.5 \pm 0.5}$ per galaxy per year, with 


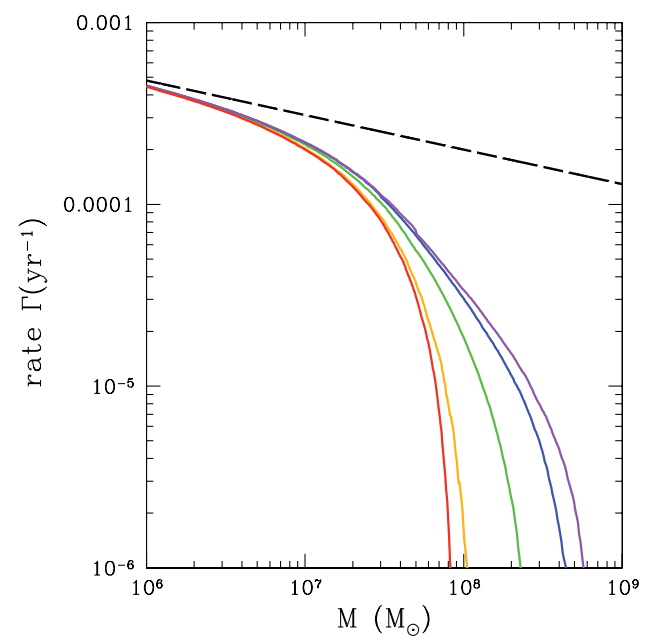

Figure 5. Tidal disruption rates for real galaxies, for a range of $\mathrm{BH}$ masses.

maximum sensitivity of the SDSS observations being to black holes with masses of $10^{7.5-8} M_{\odot}$. The SEDs of both events are fitted well by an accretion-disk model (Strubbe \& Quataert 2009; Lodato \& Rossi 2011), as shown in Fig. 6. Those fits imply that, as observed, $L_{\mathrm{bol}}>10^{45} \mathrm{erg} \mathrm{s}^{-1}$ for both flares. The flares thus satisfy a critical requirement of the proposal by Farrar \& Gruzinov (2009) that TDEs can be the sources of ultra-high-energy cosmic rays (UHECR), namely that the bolometric power of a flare that accelerates UHECRs must be at least $10^{45} \mathrm{erg} \mathrm{s}^{-1}$. Combining the rate of TDEs with their potential UHECR power shows that TDEs appear to fit the bill perfectly as accelerators of UHECRs.

TDE1: $\quad \mathrm{MBH}_{\text {fit }}=210^{7} \mathrm{M}_{\text {sun }}$

TDE2: $\quad \mathrm{MBH}_{\text {fit }}=610^{7} \mathrm{M}_{\text {sun }}$
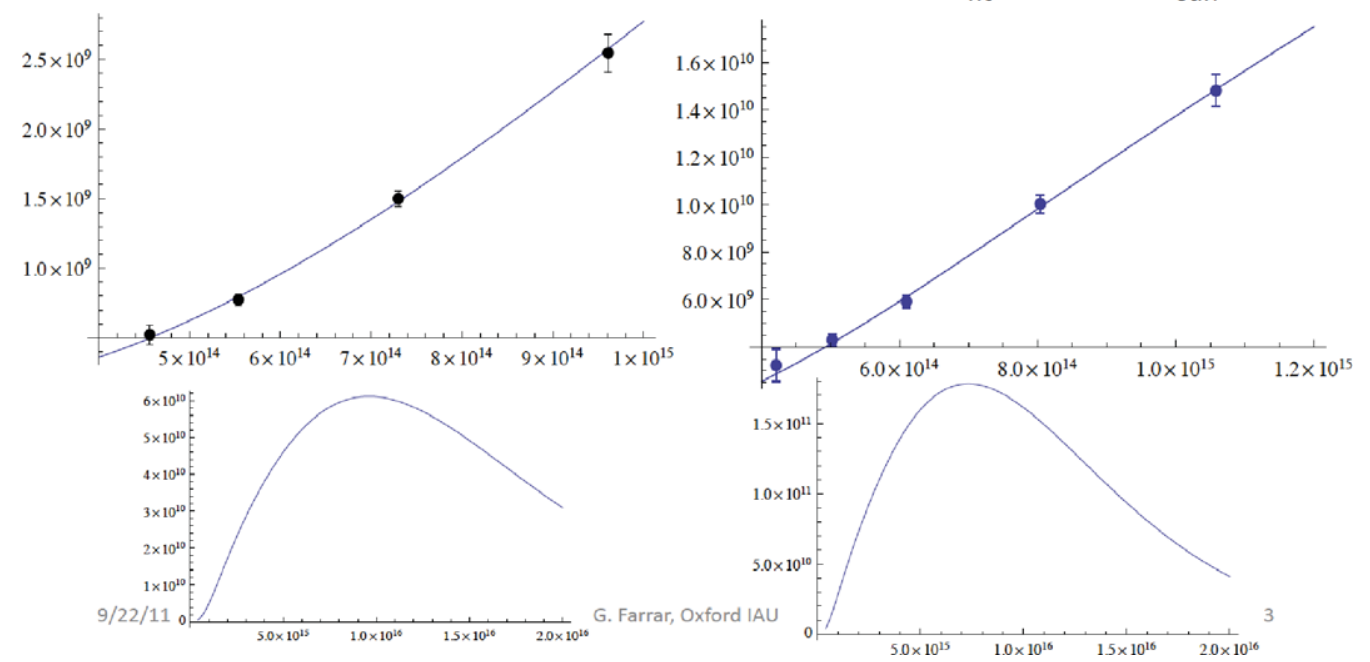

Figure 6. Fitting an accretion-disk model to the SEDs of the two TDEs from SDSS. 


\section{Summary}

The radio, optical and X-ray evidence points strongly to the interpretation that Swift $\mathrm{J} 1644+57$ is a tidal disruption flare seen in "blazar" mode as opposed to "normal accretion" mode as for the SDSS TDFs (Bloom et al. 2011; Zauderer 2011; Levan et al. 2011). A similar, unusual flare in June, Sw J2058+05, may be a second example (Cenko et al. 2011). Complementing these two dramatic events, in which we are most likely seeing emission from a recently-formed jet, are two optically-detected flares found in SDSS archived data, whose SEDs and lightcurves are well described by emission from an accretion disk and which would be very difficult to explain as anything but tidal disruption events.

\section{Acknowledgements}

I am is grateful to A. Zauderer and S. van Velzen for taking notes, and to all the participants who made the Workshop a great success. I also thank the Editors for their helpful suggestions with this contribution, and the co-Chairs for organizing an excellent and stimulating meeting.

\section{References}

Berger, E., et al. 2011, ApJ, in press.

Bloom, J. S., et al. 2011. Science, 333, 203

Campana, S., et al. 2011, Nature, 480, 69

Cannizzo, J. K., Troja, E., \& Lodato, G., 2011, ApJ, 742, 32

Cenko, S. B., et al. 2011, ArXiv e-prints

Farrar, G. R. \& Gruzinov, A. 2009, ApJ, 693, 329

Krolik, J. H. \& Piran, T., 2011. ApJ, 743, 134

Levan, A. J., et al. 2011. Science, 333, 199

Lodato, G. \& Rossi, E. M. 2011, MNRAS, 410, 359

Metzgerm B. D., Giannios, D., \& Mimica, P. 2011, ArXiv e-prints

Strubbe, L. E. \& Quataert, E. 2009, MNRAS, 400, 2070

van Velzen, S., et al. 2011, ApJ, 741, 73

van Velzen, S., Körding, E., \& Falcke, H. 2011, MNRAS, 417, L51

Wang, J. \& Merritt, D., 2004. ApJ, 600, 149

Zauderer, B. A. 2011, Nature, 476, 425 